Please note! This is a self-archived version of the original article.

Huom! Tämä on rinnakkaistallenne.

To cite this Article / Käytä viittauksessa alkuperäistä lähdettä:

Godskesen, T. E., Petri, S., Eriksson, S., Halkoaho, A., Mangset, M. \& Nielsen, Z. E. (2019) The culture of hope and ethical challenges in clinical trials: A qualitative study of oncologists and haematologists' views. Clinical Ethics, 2019:1, s. 29 - 38.

URL: https://doi.org/10.1177/1477750919897379 


\title{
The culture of hope and ethical challenges in clinical trials: A qualitative study of oncologists and haematologists' views
}

\begin{abstract}
We do not know how much clinical physicians carrying out clinical trials in oncology and haematology struggle with ethical concerns. To our knowledge, no empirical research exists on these questions in a Nordic context. Therefore, this study aims to learn what kinds of ethical challenges physicians in Sweden, Denmark and Finland $(n=29)$ face when caring for patients in clinical trials; and what strategies, if any, they have developed to deal with them. The main findings were that clinical cancer trials pose ethical challenges related to autonomy issues, unreasonable hope for benefits, and the therapeutic misconception. Nevertheless, some physicians expressed that struggling with such challenges was not of great concern. This conveys a culture of hope where health care professionals and patients uphold hope and mutually support belief in clinical trials. This culture being implicit, physicians need opportunities to deliberately reflect over the characteristics that should constitute this culture.
\end{abstract}

Keywords: Clinical trials, Ethics, Cancer, Oncology and haematology, Physicians, Informed Consent 


\section{Introduction}

Almost all clinical cancer centres carry out clinical trials. Care and research are there closely intertwined, even though the dominant paradigm of research ethics has made a sharp distinction between the two. ${ }^{1}$ c.f. ${ }^{2}$ This can be viewed as problematic: "Research ethics is characterized by a basic incoherence: on the one hand, clinical research is seen as ethically distinct from medical care; on the contrary, the obligations of investigators, especially in clinical trials, are thought to be grounded in the ethics of the physician-patient relationship". ${ }^{3}$ c.f. ${ }^{4}$ From the physicians' perspective, the primary focus is the well-being and interests of the individual patient. From the researchers' perspective, it is imperative to follow the research protocol strictly to ensure the research generates knowledge for the benefit of future patients. A physician's loyalty can fall on either side. One more inclined towards research for developing better therapeutic options may feel that her duty lies more on that side, while another colleague instead prioritises patient care, ${ }^{5}$ thus their roles might clash and tension and dilemmas emerge.

Clinicians frequently face ethical situations or dilemmas. ${ }^{6,7}$ By definition, an ethical dilemma involves the need to choose from among two or more morally preferable options or between equally unacceptable courses of action, where one choice prevents selection of the other. ${ }^{8}$ As ethical challenges are not always appropriately described as dilemmas, it might instead be preferable to speak of ethical challenges or issues. Ethical challenges can arise because of conflicting roles, each role connected with professional ideals, aspirations and norms, which might conflict in certain situations. There is thus a change of focus, from the rational conflicts involved with ethical dilemmas, to the lived conflicts resulting from particularistic and role-based circumstances in lived experience. Sometimes challenges arise because of physicians simultaneously having several different roles that make conflicting demands upon them. They can simultaneously function as clinically responsible practitioner and researcher, as well as having complementary roles such as the administratively and economically responsible, or being a collaborator with other staff categories. In their various roles, different moral, organisational or professional expectations and ideals might be present. ${ }^{9}$ For example, while clinical work often is based on an ethics of care, research is ultimately based on the value of knowledge: "If there is one overriding ethic in research, it is to learn and to tell the truth". ${ }^{10}$

We do not know much about if, and if so, how physicians carrying out clinical trials struggle with ethical concerns. To this end, we need to investigate experiences of physicians recruiting patients to clinical cancer trials 
and dealing with the sometimes-disparate frameworks of care and research. No previous empirical research has addressed these questions in a Nordic context, to our knowledge.

This study aims to find the kinds of ethical issues and challenges physicians face and experience when they include and care for patients in clinical trials; and what strategies, if any, they have developed to deal with these ethical challenges.

\section{Methods}

This study used a descriptive design and explorative qualitative approach. ${ }^{11}$ An exploratory-descriptive design is suitable as this design describe a largely unknown phenomenon in a Nordic setting from the perspective of the participants. The design is in line with the aim, to develop a rich understanding of physicians' challenges and experiences when including and caring for patients in clinical trials. In this way, lived experiences of physicians have been sought and analysed.

\section{Settings and participants}

The inclusion criteria were to work at a cancer clinic and to be involved in clinical trials at that clinic. Initially, the division heads at each Nordic university hospital, located in large urban cities, were asked for permission to perform the interviews. The intention was to recruit the same number and types of clinics in every country. After approaching the department heads, we eventually got access to two clinical research units in Sweden, one in Denmark and two in Finland (from two different hospitals). However, we did not succeed in recruiting any clinics from Norway in the timespan available.

The interviews were performed by all the authors except MM and SE. In total, 29 in-depths interviews were carried out with physicians from the Nordic clinics. Of these, two-thirds of the sample was female. The participants were at different stages of their careers (from junior resident with one year of experience to a senior with 40 years). About two-thirds were senior residents. Thirty-eight percent had familiarity with phase I trials, $59 \%$ with phase II and $93 \%$ with phase III, so there was a good spread of experience in different phases of research. Sample characteristics are presented in Table 1.

\section{[Insert Table 1 here]}




\section{Data collection}

The interview guide was semi-structured, informed by previous research, and covered known ethical issues of concern to physicians. By being largely open, it created lot of opportunities for the physicians to bring forth their own experiences, concerns and thoughts. We first compiled, discussed and wrote the interview guide in English, so that we could agree on the content and wordings. Translation into each national language ensued, in order for us to carry out the interviews in each native language. This process seemed to ensure both coherency and fascilitate feasability. The guide started with a broad question: "What does it mean for you to work with care and research?" The guide had three sections: 1) experiences when recruiting; 2) ethical dilemmas and issues; and 3) potential strategies. After each question, when necessary, follow-up questions were asked, of the form; 'Can you tell me more; When, why and how?"

A pilot study with three health care professionals was performed and to refine the interview questions we did several minor revisions. The interviews took place at the clinic or outside the hospital according to the subject's wishes and needs. They were about an hour long, audiotaped and transcribed verbatim in the local language.

\section{Data analysis}

A qualitative, inductive content analysis was used, ${ }^{12}$ as we had little former knowledge about this issue in a Nordic context. The inductive analysis was performed in three stages: preparation, organising and reporting. In the first stage, TG and SE prepared and independently coded the first six Swedish interviews. In the second stage, the study group met in a workshop and decided on which codes to use. A first draft of possible themes was identified in the same six interviews. Next, all the Swedish and Danish interview transcripts were read repeatedly, and codified by SE. The Finnish analysis was done by AH and MP according to the initially codes formulated in the workshop; the quotes considered were translated into English to facilitate further processing by the whole group. Then, TG and SE analysed the whole data set and considered categories to organize the content (using ATLAS.ti 8). We revised the categories in several steps, using Skype and frequent email exchanges, until the findings found broad consensus. When analysing and sorting the codes into categories, we could notice consistent and overarching themes in the data. Eventually, the content of the interviews was summarised into themes. 


\section{Results}

The results describe the physicians' experiences of ethics in clinical research according to three dominant themes: 1) research in support of clinical work, 2) the culture of hope, and 3) lacking strategies. Three categories were identified regarding the culture of hope: i) challenge of autonomy, ii) unrealistic hope and therapeutic misconception (TM), and iii) avoiding the talk. The quotes' provenance is indicated by S for Swedish, D for Danish and F for Finnish interviewees (numbers do not correspond to the order of interviews to ensure further anonymity).

\section{Research in support of clinical work}

When asked if they experience tensions or conflicts of interest being both clinician and researcher, many mentioned that they see the roles as associated: "These are two sides of the same coin" (D6). The research was looked upon as a necessary, integrated part of cancer care and as a condition for being able to offer patients cutting-edge treatments. Research is a natural constituent of an academic cancer centre's daily work, and benefits the patients:

We do work with studies all the time; as clinicians. It is part of being a physician at an academic hospital so then you aren't a researcher. You are just a clinician doing studies. Then you do not ponder ethical issues so much, someone else did that, you just do your part. (S1)

We then get access to drugs we never would have had otherwise. And that is good for the patient, so we do it. It is a win-win situation for the patient who gets something and the drug companies who learns something. And so we do the in-between work, so to speak. And we are happy to do it, for the patient's sake. (S3)

The roles of researcher and clinician could clash in some situations. A few physicians admitted they sometimes "sell" studies to patients, focusing on the research agenda, but most described themselves as primarily being gatekeepers or risk watchers, putting their clinical responsibilities first when responsibilities clash. For example, this entailed recommending patients to say no to participation (S4), or informing patients in such a way that emphasis is put right, according to risk: 
...it is alright to say that this is a study, it is voluntary to participate, all the things you are expected to say and do say; but there is a difference between saying that and then say 'You can also decline'. That can be said in an appropriate way, you can put more or less emphasis on it. (D16)

Other examples of gatekeeping mentioned were finding a protocol so unfruitful that staff hesitates to include patients in it, and cases where the experimental arm offered less treatment than the standard arm:

For example, in a study where you receive three months treatment instead of six months standard treatment, you need as a physician to be very sure that this is least as good before participating. However, every time you inform a patient for such a study, it feels problematic, because the patient often asks the difficult questions: Are you sure that this is just as good? And so on... Then you cannot say ... I mean, if you are sure, you wouldn't do the study, that's the problem. All research aiming to reduce treatment intensity is thus ethically problematic by definition. (S4)

Thus, physicians expressed ambiguity. One view was that drug trials are good for both patients and drug companies; but patients asking difficult questions about the efficacy of the study drug could sometimes challenge this view.

\section{The culture of hope}

The ethical challenges brought up most by the physicians were the challenge of autonomy, unrealistic hope, TM and the pull to avoid difficult discussions with the fatally ill patients.

\section{The challenge of autonomy and informed consent}

A critical ethical challenge identified concerned patient autonomy. Most of the physicians said that explaining clinical trials to patients is very demanding. A majority was concerned about how much information patients really do understand. Even physicians sometimes have difficulties with understanding the trial protocols, they believed: 
These likelihood things are like UFO things for most people. People don't understand relative and absolute benefit or harm, or difference between them. You must explain in words of one syllable. (F3)

We also [physicians] have difficulties in understanding exactly how they work, and why they are not working, i.e. dose escalation studies; doctors hardly understand the point of them, so patients just can’t. (D1)

Patients, it was noted by one physician, sometimes get informed about the same drug twice, first due to their treatment and then again when being enrolled in a trial. The varying level of detail about possible adverse effects can make patients worried: "But, are you usually hiding something from us, as it is stated like this here?" (D8)

Many interviewees found the written information about the study of little help to patients. The information is too long, complicated and include excessive facts about side effects: "And that I find annoying, there is like some sort of barrier; you seemingly just can't distribute patient information which can be easily understood, not looking like a telephone directory" (D11). When asked if they believe that the patients read the written information provided, many physicians were unsure. Great help with these difficulties from the nurses were often emphasized; they could be relied on to elaborate and deepen study understanding when physicians felt a bit unsure.

I think our nurses are very good at talking with relatives. The clinical trial unit has great skills; they genuinely look to the individual patients and make sure they are well informed. (D7)

Another major concern was that patients often want to enrol in all kinds of research to obtain cutting-edge drugs not part of conventional therapy (such as immunotherapy): 'You can get patients to sign up for anything' (D8). Phase I trials were mentioned by most physicians as the major ethical challenge:

When one is to include patients in a phase-I-study investigating a drug, well, the purpose of a phase-1-study is to investigate toxicity. You are not interested in efficacy; you just want to check 
that the very drug does not kill the patients. Patients usually enrol because they want something, like a straw. (S1)

Some other types of studies can be difficult to inform about as patient interest is lacking:

To me, they seem much more interested in hearing about studies involving treatment. Studies without any extra pain, side effects, medicines, somehow being exposed to something, scans for example, they are not that interested in, I believe. They prefer to focus on that for which they came. (D17)

This tendency to reach out for the latest innovative drugs and treatments was often influenced by exposure to news media or the internet, it was said:

The press and the internet is where they often find out all sorts of things, which still is in development, but where much knowledge is disseminated underway. And then, as they learn about it, they can't understand why this treatment isn't publicly available. (D8)

Some interviewed described how relatives sometimes want to control the decision. Relatives could even put pressure on the health care personnel to try and make sure the patient was put in the experimental arm when randomised:

Then this person says to me, "you make sure he gets in the right arm". So he tried to push us into ... influencing the result of randomization, which we cannot do. I mean, he does not understand this, but we can't do it. But it came to a huge conflict with this son when I said I couldn't do it so "take it or leave it". (S3)

\section{Unrealistic hope and therapeutic misconception}

Unrealistic hope and/or the therapeutic misconception were mentioned in all interviews, particularly encountered in phase I trials. This can be a challenge: “[A] patient can have hope, but you must be realistic. You cannot cheat patients" (F2). When asked if patients often have unrealistic hope, one respondent said: "Yes, they always have. 
That is part of the game. However, unfortunately, the sickest patients are worst in this respect" (S4). Another said: "[A] large portion of patients think they will be cured from the incurable disease" (F3). When asked if they believe that patients in end of life will participate in all kind of trials related to hope, one physician answered: "I believe that if you were to ask all fatally ill cancer patients, a truly substantial part would say that they want to participate precisely because of that hope..." (D14).

Many interviwees stated that patients truly believe that in research patients' best interests are promoted and that patients therefore have difficulties to distinguish clinical research from standard treatment: "They believe that if the clinic offers a new treatment, it is because we suppose it's better" (D16). This, as several physicians pointed out, were not only an issue among the patients but also for health care personnel:

We do believe there is a straw to reach for as long as we include patients in studies; this might, by all means be unrealistic, but in such cases, also the clinical judgment is that there must be a chance; otherwise, the patient would not be included in the study. (S9)

Many physicians reflected over their belief that often it is beneficial for patients and relatives to have hope, even if unrealistic: "It may not be realistic, but it gives them some hope in the meantime. And I think that's an advantage." (D16). Some stated that hope must be seen as a basic condition of life. Quite many said that participation in clinical trials is in the best interest of the individual patient and that they therefore could push clinical trials as an option: "I believe we cross the line a bit for the individual, but also that we do so out of benevolence somehow" (S7).

\section{Avoid the talk}

Breaking bad news was highlighted as a complex and challenging task which sometimes were avoided: “...as long as I haven't spoken; it's not there" (D1). As participation and hope was seen as a patient strategy to keep going through hard times, it became a reason for physicians to not deliver bad news to patients, trying to be supportive. All agreed that maintaining hope is an important and vital task for health care professionals, and for some of the physicians the easiest thing to do was giving end of life patients a straw to clutch: “...it is better just to say 'let's try this" (D1). 
...initially one tries to avoid being unrealistic but one doesn't present patients with a worst-case scenario..."we'll see how this works", and then: "But will not all get cured?" Then one has to answer that no, you won't. But it might go well for a long time and then...one has to downplay it so that...deep inside they all know they are dying of cancer, they know that, so they want me to say: "this new treatment will cure you!". I do not say that but often rather that we'll see how it goes... and hopefully it does go in the right direction. (S3)

\section{Lacking strategies}

Many physicians said they lack forums for addressing ethical questions and that there is no clear strategy for dealing with ethical challenges. On the other hand, most physicians insisted that their clinic displays acceptance of and openness to ethical questions and discussions. When asked why they then do not explicitly discuss ethical issues, one explanation was: “I just don’t believe we think we have ethical dilemmas at all. So it is usually just not brought up" (D1). Others said it was related to poor time resources or logistical hindrances: "We have few discussions about ethical dilemmas. We have a shortage of time, and therefore we don't have time for such meetings. It's a logistical hindrance in daily basis" (S6). Another said: "[We talk] less and less because the time we have together is decreasing" (D5). Time pressure was mentioned in almost all interviews as an essential barrier for addressing ethical challenges. Still, the self-image was of doctors having the individual craft and will to decide the best way to solve the problem. As one stated:

...we do talk about it, but as with many doctors, we are very autonomous people, that is, we do what we believe is best. Therefore, just because some others think this or that, well, there is no guarantee that the assigned physician or the one at the bedside chooses to comply. (D7)

Many said they had to deal with ethical challenges by themselves but help from colleagues were also sought. For example, they would call a close and trusted colleague: "The first thing to do then is probably to call a close colleague right away and say - what would you do here?" (D2). An alternative was to talk about troubling situations over a lunch or coffee, on an ad hoc basis: "We can debrief and talk it through over lunch" (S8). Several of the interviewees mentioned that collaboration with research nurses was necessary. Such collaboration was important for the physicians when finding it a conflict between patient autonomy and poor health, or when 
being unsure about patient consent. In such cases, the physician and the nurse could reflect together and act for the patient's good, hopefully.

Yes, I probably discuss those [ethical issues] more with nurses than with my physician colleagues. I do not want to generalize, but nurses has a keener eye ... and it is good that some has that perspective, as physicians might be more concerned with treatment issues ... My experience is that nurses sooner will put an end to some treatment or so, or not include [patients] ... while we doctors want to treat and maybe, sorry if this sounds a bit harsh, can see things from a greater perspective. That it is good for medicine, not just the individual person. (D14)

Among some physicians, individual strategies could be found. One mentioned that when some patients after protocol information said no to participate, the very existence of this 'no' became a sign that the process of information giving actually worked. If every patient asked had said yes to take part in a trial, there would be a concern. Another physician employed a more rational approach when encountering ethical challenges:

I do a lot of rationalizing. Actually I might have considered a few cases being wrongly handled, but most often I think that this was the option available; we couldn't do otherwise. You tend to become desensitized. This does not have to be a bad thing; you should be diligent but not too sad. It is for the patient and the next-of-kin to be sad; the physician should be rational. (S8)

In general, the physicians relied heavily on ethical review boards or the study protocol itself to take care of the ethical challenges beforehand. They reasoned that if the trial had had many ethical concerns, the ethics committee would not have approved it. When patients in e.g. phase I studies are too sick to be included or need to be excluded because of physical deterioration, many physicians thought that inclusion/exclusion criteria proved helpful and could be relied upon to work out the ethics by itself:

But there are exclusion criteria in the trials that make [some patients] unfit to be included when unethical, as, I believe those who have designed the study have thought about which groups are badly suited for it, which then is nicely and clearly expressed in exclusion criteria. That goes so well together with how unethical it would be to include the group in a trial. (D2) 
So, ethical issues were often downplayed. Ethical issues were nevertheless present under the surface. The physicians could come up with some strategies to handle ethical challenges: a prominent one was discussions with nurses (more so than with other physicians).

\section{Discussion: The culture of hope}

A common outlook was the close relationship between research and care. The respective goals of these activities were not perceived to be in opposition; on the contrary, the overriding idea was that they coincide. Strikingly, many physicians downplayed the importance of ethical problems related to their daily research work. We will look at the major ethical challenges identified, before discussing how many findings indicate the presence of a 'culture of hope', where potential ethical challenges might often go unnoticed (Figure 1).

Ethical dilemmas resulting from moral doubt or uncertainty, i.e.the need to choose among two or more morally acceptable options or between equally unacceptable ones, were not mentioned often. Most ethical challenges were related to autonomy, an unreasonable hope for benefits, or the therapeutic misconception (particularly in phase I trials), findings in line with other studies. ${ }^{13}$ Physicians were concerned over patient ability to understand the aim and scope of trials, especially phase I trials, as many patients are willing to participate in anything that offer a glimpse of hope. They do not show much interest in studies without the potential to benefit them. This makes informing patients difficult, and the physicians often seemed to experience a moral duty to respect autonomy and make sure patients decide whether to participate from a proper understanding of the study. But regardless of whether you inform about a study that a patient can frame in terms of hope or about a study lacking that quality, the information will not be just neutrally received. The understanding of the information is rather heavily shaped by patient interests, which many physicians in the study seemed well aware of. Such a willingness to participate in experimental research is previously well documented, as well as poor understanding of trial purpose. ${ }^{14-17}$

Unrealistic hope was a prominent ethical issue reported. The physicians felt concerned both with patients losing touch with what was going on, and with their own part in creating or sustaining unrealistic expectations on possible benefit from trials. One possible explanation for unrealistic hope stem from patients not understanding the difference between care and research and then mistakenly believing that research aims primarily for their 
benefit (the therapeutic misconception). Factors facilitating such unrealistic hope include having the research carried out by one's own physician, ${ }^{18}$ which was commonly occurring among the physicians in our study. Joffe and Weeks ${ }^{19}$ have shown that not only patients but also physicians and investigators might share such misconceptions of clinical trials. ${ }^{20}$ This is further discussed by McDougall et al., ${ }^{21}$ who suggest a new concept, therapeutic appropriation (TA). TA occurs, they state, when "patients, or clinicians, actively reframe research participation as an opportunity to enhance patients' clinical care, while simultaneously acknowledging the generalised research aims". Then they do not mistakenly conceptualize research as care, but are seizing an opportunity to find hope in something they know is research. This strikes us a valid interpretation also of our data.

Despite identifying these ethical challenges, most physicians stated that struggling with them was not a great concern. If they sometimes had some pressing ethical issues of this kind they might discuss them with nurses or in ad hoc meetings with colleagues, but in everyday work, such challenges were usually not brought to the fore. Looking at the results, several reasons may explain why these ethical issues are not given more attention.

\section{[Insert figure 1 here]}

First, there simply is no time to attend to ethical issues and no institutional systems in place to deal with them. ${ }^{22}$ All the participants agreed on this, basically, and expressed that clinical work and study protocols are in strong focus. It is not surprising then that ethical issues are out of view. This phenomenon, called "ethical fading", has previously been studied: when we focus on other aspects of a situation, this psychological process make the ethical aspects of a decision disappear from view. ${ }^{23}$ As the number and complexities of clinical guidelines typically are overwhelming for physicians,${ }^{24}$ striving for compliance with just those policies and guidelines will leave little room for thinking about research ethics.

Second, physicians supposed that any ethically difficult issues were already taken care of by those responsible (the PIs) for the study, by the physician who refers patients to the specific study and by the Ethical Review Board (IRB or ERB). Since the ERB had previously evaluated the ethics of the clinical trial protocol and its methods, and appropriate steps had been taken to protect the rights and welfare of patients, they were at ease with the protocol. Some mentioned explicitly that the inclusion and exclusion criteria could be of good help 
when dealing with ethical issues, such as unrealistic hope. In this way, the physicians relied on the ERBs and protocols to do the ethical work for them; so for e.g. when patients in an advanced stage of cancer are in poor clinical condition but still want to participate, making use of the exclusion criteria were thought to be an aid in protecting them from harm.

Third, most physicians were convinced that in general it is an advantage to participate in clinical trials. Physicians felt no moral conflict in endorsing enrollment as an important option, mostly because they were convinced that they met patient interests by offering access to the best available treatment. This positive outlook even extended to phase I trials for some of the physicians, but most of them pointed to indirect benefits in that setting, such as meeting patient wishes for more surveillance or contact. It is important to bear in mind, however, that most experimental drugs in phase I trial are not effective. ${ }^{25}$ Also, there is often no substantial therapeutic difference between the standard treatment and the clinical trial drug. ${ }^{26,27}$ In this study, some physicians were convinced research constitutes the best available treatment for patients, and when becoming uncertain whether it is so in a specific case, they acted as gatekeepers for patient enrolment, as a physician's primary focus is the benefit and interests of the individual patient. This finding is consistent with the literature. In the study of Joffe and Weeks ${ }^{19}$ U.S. oncologists $(n=547)$ were asked to state the aim of clinical trials and to specify why they asked patients to participate. Most of them said that clinical trials aim to collect generalizable knowledge for future research; still, many also claimed that a principal purpose is to benefit them. Some oncologists viewed trial participation as a state-of-the-art therapy. This shows that there is a lack of consensus among physicians "working with care and research about the nature of the relationship between research and treatment in clinical trials". 27

The end result is a culture of hope where health care professionals and patients perpetually reinforce their common belief in clinical trials and their hope for something that might be somewhat helpful in a dreadful situation. Whether such therapeutic appropriation is a good thing or not is not for us to say. The involved do certainly not embrace this process consciously; but it is a result of several factors, reflecting deep and common human traits and dispositions. What is troubling is the very fact that this culture is not the result of weighing moral aspects of the culture. Is it better to have hope than having to realize that the end is near? Is being truthful more important than being compassionate for a doctor? Is doing what you want in the final days more valuable 
than being "a fighter to the end"? Some such questions are deeply personal, some are professional rather, while some belong on a societal level, but if they fade from view they cannot be thought over at all.

From the perspective of research ethics, a demarcation between medicine and research has been widespread. This distinction was explicit in codes and regulations such as the Declaration of Helsinki and the Belmont Report, for example. In research there are partly different norms, meant to protect subjects from the kind of abuse that historically have shaped and provoked the emergence of research ethics. Therefore, in a way, a culture of hope situated in a care setting clashes with some of the traditional principles of research ethics that is built upon such a distinction. This has possible consequences for the conduct, review, and regulation of clinical trials. This is not least so when it comes to addressing the issue of how to present the information to the patients. ${ }^{28}$ Detailed regulations for trials exist under the supervision of ethical committees or institutional review boards, while a culture of hope might to a larger extent leaving many issues to professional discretion and judgment. True, in some countries, the existence of clinical, hospital or institutional ethics committees might present an opportunity for ethical deliberation beyond professional self-governance, but they are not that established in the Nordic countries. At the same time, there is in the practice of medicine often no sharp separation between practicing medicine and conducting research nowadays and many stakeholders argues for new ways of integrating the two. For example, a Learning Healthcare System is defined by the Institute of Medicine as a system in which "science, informatics, incentives, and culture are aligned for continuous improvement and innovation, with best practices seamlessly embedded in the delivery process and new knowledge captured as an integral by-product of the delivery experience" ${ }^{28}$ In this line of thought, a culture of hope might still be preferable to one where people for ethical reasons must be deprived of their own human impulses and motivations - not least as research collaboration between science and society should be based on a common understanding and commitment to shared goals and values. ${ }^{29}$ The issues found here are highly complex, where patients and physicians have a variety of motivations and emotions affecting choices and attitudes, which means there are no easy answers.

In conclusion, the present system or culture needs to become visible to those working in it and be openly discussed. Ethics training with a focus on normative education and visible formal systems may only be partially effective. These issues also includes various hidden, informal, systematic factors of communication, surveillance, and sanctioning mechanisms that together make up an organizational climate or ethical 
infrastructure. ${ }^{30}$ There is a need to provide many more opportunities for physicians to come together for reflection and conversation around these issues - an infrastructure that facilitates and promote ethical reflection. ${ }^{31}$ Ethical challenges should not be allowed to fade away from sight, but be put high on the agenda for a future where care and research become even more closely knit and a likely place where we put our trust and hope for a future.

\section{Strength and limitations of study}

The current study adds to previous work by investigating the views and experiences of physicians in three different clinical cancer settings within the Nordic countries. The key strength of this study is the large sample size; physicians had a significant variety of both short and long experience of clinical trials, in addition to having experiences from all three trial phases. However, these findings have some limitations. Although the sample size was large, the small sample size in each country means it is not possible to draw conclusions on any differences between the countries. It is up to the reader to determine to what extent the results are transferable to settings outside that which was studied here. ${ }^{32}$ Nevertheless, the findings from Sweden, Denmark and Finland are not unique to this context and are therefore likely to be representative of other clinical trials setting outside the Northern countries.

\section{Ethical considerations}

This study followed each country’s regulatory demands. According to Swedish, Danish and Finnish law on research involving humans, no ethical approval is required for this kind of non-interventional study which does not involve risks or the processing of sensitive personal data. ${ }^{33}$ Approval to perform interviews was obtained from the department head of each hospital. The study followed the requirements of the Declaration of Helsinki,

${ }^{34}$ in that participants were informed about the study, the handling of personal data, and how confidentiality would be kept in the management of material and in publishing and presenting results ( $\S 24-26)$. Written informed consent was obtained at the start of the interviews. All identifiers were removed from transcripts before being distributed to the research network. 
Rhodes R. Rethinking research ethics. Am J Bioeth. 2010; 10: 19-36. HHS. The Belmont Report: Ethical Principles and Guidelines for the Protection of Human Subjects of Research. Department of Health, Education, and Welfare., 1979. Joffe S and Miller FG. Bench to bedside: mapping the moral terrain of clinical research. Hastings Cent Rep. 2008; 38: 30-42. Katz J. Human Experimentation and Human Rights. Saint Louis University Law Journal. 1993; 38: 7-54. Nardini C. The ethics of clinical trials. Ecancermedicalscience. 2014; 8: 387. Brown RF, Butow PN, Butt DG, Moore AR and Tattersall MH. Developing ethical strategies to assist oncologists in seeking informed consent to cancer clinical trials. Soc Sci Med. 2004; 58: 379-90. https://doi.org/10.1016/0277-9536(92)90169-Q Hurst SA, Perrier A, Pegoraro R, et al. Ethical difficulties in clinical practice: experiences of European doctors. J Med Ethics. 2007; 33: 51-7.

Ong WY, Yee CM and Lee A. Ethical dilemmas in the care of cancer patients near the end of life. Singapore Med J. 2012; 53: 11-6.

Taylor KM. Integrating conflicting professional roles: physician participation in randomized clinical trials. Soc Sci Med. 1992; 35: 217-24.

Emanuel EJ. The Oxford textbook of clinical research ethics. Oxford ; New York: Oxford University Press, 2008, p.xx, 827 p.

Polit DF and Beck CT. Nursing research : generating and assessing evidence for nursing practice. Ninth Edition. ed. Philadelphia: Wolters Kluwer Health/Lippincott Williams \& Wilkins, 2012, p.xiv, 802 pages.

Elo S and Kyngas H. The qualitative content analysis process. J Adv Nurs. 2008; 62: 107-15.

Ulrich CM, Zhou Q, Ratcliffe SJ, Ye L, Grady C and Watkins-Bruner D. Nurse Practitioners' attitudes about cancer clinical trials and willingness to recommend research participation. Contemp Clin Trials. 2012; 33: 76-84.

Beadle G, Mengersen K, Moynihan S and Yates P. Perceptions of the ethical conduct of cancer trials by oncology nurses. Eur J Cancer Care (Engl). 2011; 20: 58592.

Dolly SO, Kalaitzaki E, Puglisi M, et al. A study of motivations and expectations of patients seen in phase 1 oncology clinics. Cancer. 2016.

Godskesen T, Nygren P, Nordin K, Hansson M and Kihlbom U. Phase 1 clinical trials in end-stage cancer: patient understanding of trial premises and motives for participation. Support Care Cancer. 2013; 21: 3137-42.

Mangset M, Forde R, Nessa J, Berge E and Wyller TB. I don't like that, it's tricking people too much...: acute informed consent to participation in a trial of thrombolysis for stroke. J Med Ethics. 2008; 34: 751-6.

Appelbaum PS, Lidz CW and Grisso T. Therapeutic misconception in clinical research: frequency and risk factors. IRB. 2004; 26: 1-8.

Joffe $\mathrm{S}$ and Weeks JC. Views of American oncologists about the purposes of clinical trials. J Natl Cancer Inst. 2002; 94: 1847-53.

Miller FG, Rosenstein DL and DeRenzo EG. Professional integrity in clinical research. JAMA. 1998; 280: 1449-54.

McDougall R, Martin D, Gillam L, Hallowell N, Brookes A and Guillemin M. Therapeutic appropriation: a new concept in the ethics of clinical research. J Med Ethics. 2016; 42: 805-8.

Rahman S, Majumder MA, Shaban SF, et al. Physician participation in clinical research and trials: issues and approaches. Adv Med Educ Pract. 2011; 2: 85-93. 
23. Tenbrunsel AE and Messick DM. Ethical Fading: The Role of Self-Deception in Unethical Behavior. Social Justice Research. 2004; 17: 223-36.

24. Carthey J, Walker S, Deelchand V, Vincent C and Griffiths WH. Breaking the rules: understanding non-compliance with policies and guidelines. BMJ. 2011; 343: d5283.

25. Hay M, Thomas DW, Craighead JL, Economides C and Rosenthal J. Clinical development success rates for investigational drugs. Nat Biotechnol. 2014; 32: 40-51.

26. Djulbegovic B, Kumar A, Glasziou P, Miladinovic B and Chalmers I. Medical research: Trial unpredictability yields predictable therapy gains. Nature. 2013; 500: 395-6.

27. Vist GE, Bryant D, Somerville L, Birminghem T and Oxman AD. Outcomes of patients who participate in randomized controlled trials compared to similar patients receiving similar interventions who do not participate. Cocbrane Database Syst Rev. 2008: MR000009.

28. LearningHCS. Learning Healthcare System. 2015.

29. Nurmi SM, Halkoaho A, Kangasniemi M and Pietila AM. Collaborative partnership and the social value of clinical research: a qualitative secondary analysis. BMC Med Ethics. 2017; 18: 57.

30. Tenbrunsel AE, Smith-Crowe K and Umphress EE. Building houses on rocks: The role of the ethical infrastructure in organizations. Soc Justice Res. 2003; 16: 285-307.

31. Höglund A, Eriksson S and Helgesson G. The role of guidelines in ethical comptence-building: perceptions among reserach nurses and physicians. Empirical Ethics. 2010; 5: 95-102.

32. Lincoln YS and Guba EG. Naturalistic inquiry. Beverly Hills: Calif: Sage Publications, 1985.

33. SFS. Lag om etikprövning av forskning som avser mäniskor Socialdepartementet, 2003:460.

34. World Medical Association [WMA]. Declaration of Helsinki. 2013. 
Table 1. Participants' characteristics

Physicians $(\mathrm{n}=29)$

\begin{tabular}{lr}
\hline Gender & \\
Female & 21 \\
Male & 8
\end{tabular}

\section{Age (years)}

$\begin{array}{ll}\text { Range } & 29-60 \text { years } \\ <29-39 \text { years } & 8 \\ 40-49 \text { years } & 13 \\ 50->60 \text { years } & 8\end{array}$

Working years in oncology

Range $1-40$

$\begin{array}{ll}\text { Median } & 17\end{array}$

\section{Practice setting}

Junior resident 6

Senior resident $\quad 23$

Working in research and clinic 24

Only working with research 5

$\begin{array}{ll}\text { Phase I } & 11\end{array}$

$\begin{array}{ll}\text { Phase II } & 19\end{array}$

$\begin{array}{ll}\text { Phase III } & 27\end{array}$

\section{Country}

$\begin{array}{ll}\text { Denmark } & 17\end{array}$

Sweden 9

Finland 3 